\title{
Technique training does not improve the ability of most patients to use pressurised metered-dose inhalers (pMDIs)
}

\author{
Alison Hardwella, Victoria Barber ${ }^{\mathrm{b}}$, Tina Hargadon ${ }^{\mathrm{b}}$, Eddie McKnight $^{\mathrm{b}}$, John Holmesc, \\ * Mark L Levy \\ ${ }^{a}$ National Services for Health Improvement (NSHI), Dartford, Kent, UK \\ ${ }^{\mathrm{b}}$ Magister Consulting, Bristol, UK \\ Teva UK Limited, Harlow, Essex, UK \\ ' Senior Clinical Research Fellow, Allergy \& Respiratory Research Group, Centre for Population Health Sciences: GP section, The University \\ of Edinburgh, Scotland, UK
}

Originally submitted 16th June 2010; resubmitted 21st November 2010; revised 5th December 2010; accepted 6th December 2010;

online 11th January 2011

\begin{abstract}
Aims: According to guidelines, inhaler technique should be tested in all patients, particularly those with poorly controlled asthma. We aimed to assess uncontrolled asthma patients' ability to use a pressurised metered-dose inhaler (pMDI) using the Aerosol Inhalation Monitor (AIM, (C) Vitalograph).

Methods: Practices invited patients for a detailed clinical review by trained asthma nurses according to practice-agreed protocols. Reviews took place from 1st April to 30th June 2008, and included checking of inhaler technique. Reasons for invitations included time since last review, asthma control, prescriptions, and adherence to medical advice. A proxy measure of control - the total number of short-acting $\beta_{2}-$ agonist (SABA) bronchodilator inhaler canisters prescribed in the previous 12 months - was used. Data on 77 pre-determined clinical parameters (including prescribing and healthcare utilisation data) were collected. Patient-completed postal symptom questionnaires (the RCP 3 questions) were obtained in some patients. All patients using pMDls had at least two assessments using the AlM, and where appropriate inhaler technique education was provided.

Results: 2123 (24\% of those invited) symptomatic asthma patients were reviewed; 1291 (61\%) were using pMDIs (mean age 52 years; SD 21), of whom over $80 \%$ were in BTS/SIGN Steps 2 and 3. 1092 (85.6\%) of those patients using pMDIs failed the first AIM assessment. There was a significant increase in the number of patients able to use their pMDIs correctly following instruction after the second (129 to 260 of 1197 patients, $p<0.01$ ) and third ( 61 to 181 of 528 patients, $p<0.01$ ) tests. However, $78.4 \%$ and $65.7 \%$ of those tested twice and three times, respectively, failed the AIM assessment despite instruction. Logistic regression analysis failed to show any effect of age and BTS step on these outcomes.

Conclusions: A majority of symptomatic asthma patients in this study were unable to use pMDIs correctly. It is essential to check all patients' ability to use their prescribed inhalers regularly. Cost alone should not determine prescribing recommendations.

(C) 2011 Primary Care Respiratory Society UK. All rights reserved.

A Hardwell et al. Prim Care Respir J 2011; 20(1): 92-96

doi:10.4104/pcrj.2010.00088
\end{abstract}

Keywords asthma, management, nurse, observational, primary care, treatment, pMDI, inhaler technique, education

See linked editorial by Corrigan on pg 13

\section{Introduction}

Patients prescribed pressurised metered-dose inhalers (pMDIs) require training and skill to co-ordinate both activation and inhalation of the inhaler. According to guidelines, inhaler technique should be tested in all patients, particularly those with poorly controlled asthma. ${ }^{1,2}$ In practice, it is logical that inhalers are prescribed only after patients have received training and have demonstrated their ability to use the device. Furthermore, UK national ${ }^{1}$ and international ${ }^{2}$ asthma guidelines recommend that patients have their inhaler technique assessed

\footnotetext{
* Corresponding author: Dr Mark L Levy, Allergy \& Respiratory Research Group, Centre for Population Health Sciences: General Practice Section, The University of Edinburgh, Doorway 3, Medical School, Teviot Place, Edinburgh, Scotland, EH8 9AG, UK

Tel: +44 (0)1461 600639 Fax: +44 (0)1461 207819 E-mail: marklevy@animalswild.com
} 
by a trained healthcare professional as part of a structured clinical review, since many patients ${ }^{3-5}$ and healthcare professionals $^{6}$ are unable to use a pMDI correctly, ${ }^{3-5}$ with detrimental effect on asthma control. ${ }^{4}$ Increasing age has also been shown to reduce patients' ability to use their MDI correctly. $^{5}$

This service evaluation focussed on uncontrolled asthma patients who were currently using a prescribed pMDI. They were reviewed in nurse-run clinics. We aimed to assess these patients' ability to use - and (if needed) to learn to use - a pMDI, using the Aerosol Inhalation Monitor (AIM, C Vitalograph) which tests inspiratory flow, synchronisation, and breathholding.

\section{Methods}

\section{The EACS service}

The 'Enhanced Asthma Care Service (EACS)', sponsored by Teva UK Limited, is offered to UK general practices for their patients with a confirmed diagnosis of asthma. Practices are invited to participate by Teva UK Limited sales representatives. An EACS Respiratory Specialist Nurse Advisor meets with the practice teams to discuss and agree an asthma review specification tailored to the patients' (according to practice data) needs as well as those of the practice. The service includes a detailed clinical asthma review by trained nurses (who had attained at least a recognised asthma nurse diploma which includes training in teaching inhaler technique - see www.educationforhealth.org) according to pre-agreed practice protocols based on published guidelines. ${ }^{1,2}$

The Specialist Nurse Adviser then agrees a 'Course of Action' with the general practitioners (GPs) to decide the basis for selecting patients for invitation for clinic review and the actions they would like the nurse to take according to agreed practice protocols. Factors used by practices to select patients included: time since the patient's last asthma review; current levels and types of prescriptions; current asthma control; and adherence/non-adherence to medical advice. Computer searches on diagnosed asthma patients using dedicated general practice software (MIQUEST ${ }^{7}$ ) were used to extract data on 77 pre-determined clinical parameters (including prescribing and healthcare utilisation data).

\section{Asthma control definition}

For the purpose of defining asthma control (one of the parameters used in selecting patients for invitation for review), we used a number of factors. One was a proxy measure based on the total number of short-acting $\beta_{2}$-agonist (SABA) bronchodilator inhaler canisters or units prescribed in the last 12 months. SABA bronchodilators can be prescribed as pMDIs, dry powder inhalers (DPIs), and nebuliser liquid, in the UK. Typically the pMDIs contain 200 doses, while most DPIs contain 60 to 100 doses. According to $\mathrm{UK}^{1}$ and GINA ${ }^{2}$ guidelines, an asthma patient using their reliever inhaler more than two or three times a week is deemed to be uncontrolled and in need of preventer medication initiation or review. Therefore, we assumed that it was reasonable for practices to decide to review patients who were prescribed more than four SABA bronchodilator inhaler units a year. Other factors used for determining poor asthma control included: prescribing data (e.g. short courses of oral steroids); health care utilisation; and in some cases a postal symptom questionnaire incorporating the Royal College of Physicians (RCP) '3 questions '1,8 which was sent to 'uncontrolled patients' as well as other patients identified individually by the practice for review.

\section{Patient assessment}

During the nurse clinic asthma review, patient details were summarised on a clinic proforma. Reviews took place from 1st April to 30th June 2008. All patients underwent inhaler technique assessment based on their current prescribed inhaler using two sorts of placebo device - an In-check monitor ( ${ }^{\mathrm{TM}}$ Clement Clarke International), and for those using pMDIs a Vitalograph Aerosol Inhalation Monitor (AIM). 9,10 The AIM measures inspiratory flow, ongoing flow, and breathholding. A colour-coded response indicates the quality of the patient's technique (green for good technique). A green band is displayed for each of the following:

- Inspiratory flow of between 10 and $50 \mathrm{~L} / \mathrm{m}$

- Flow of between 10 and $50 \mathrm{~L} / \mathrm{m}$ for at least $50 \%$ of the next 3 seconds.

- Breath hold at end of inspiration of at least 5 seconds.

The flowhead (dummy pMDI) is calibrated to the instrument. All flows have a tolerance of $+/-5 \mathrm{~L} / \mathrm{m}$ (personal communication, Vitalograph).

All patients previously prescribed and currently using pMDls had at least two assessments using the AIM. They received inhaler technique education if they failed on any of the three AIM measures. Education was focused on the AIM measures that the patient failed to achieve, using the suggested methods detailed in the manufacturer's patient information leaflets as well as detailed instruction methods learnt by the nurses as part of their diploma training. Any changes to medication were implemented by the nurses according to the pre-agreed practice protocol. Following clinic assessment, all patients were discussed in detail by the nurses with the GP who decided on future management.

All information processed was solely for the purpose of carrying out the service, and complied fully with the Nursing \& Midwifery Council (NMC) Code of Conduct (Nursing Staff), ${ }^{11}$ the ABPI Code of Practice 2008, ${ }^{12}$ Caldicott Principles, ${ }^{13}$ and the UK Data Protection Act 1998. ${ }^{14}$

All identifiable patient data remained in the practice, along with any practice reports generated. As this EACS service was provided by NSHI (National Services for Health Improvement) at the request of practices for the benefit of their own patients, and 
in accordance with agreed practice protocols, ethics approval was not deemed necessary.

\section{Data analysis}

The results of the inhaler technique testing using the AIM machine were analysed by comparing the success or failure of the test using the Chi-squared statistic. T-test was used to determine the relationship between the number of inhalers prescribed and selection for patients' clinic invitations (which was based upon their proxy measure of control).

\section{Results}

Having agreed protocols, each participating practice invited patients to attend the clinics. At the time of writing, 100 practices caring for about 0.5 million patients, including 30,779 diagnosed asthmatics (prevalence 6.9\%), have used the EACS service.

There was evaluable data available for 21,647 (base population), and $8843(41 \%)$ were chosen by the practices according to their various inclusion criteria and were invited to attend the clinics. 2123 patients (24\%) attended for review between 1st April and 30th June 2008. The attending patients' mean age was 52 yrs (SD 21; 48\% under 45 years; 42\% 4675 years), slightly older than the base population (mean age 41 yrs, SD 23; $80 \%$ over 17 years old). In the invited patients, the number of prescriptions per patient in the previous 12 months for SABA inhalers ranged from $0-108$ inhalers of $60-200$ doses, with $50 \%$ being prescribed four or fewer SABA inhalers in the previous 12 months.

There was an association between patients BTS/SIGN treatment step ${ }^{1}$ and whether they were invited to attend; of the patients who were not invited, more were in treatment steps 0 and 2 ( 69 and $62 \%$, respectively), while more patients in all the other steps $(1,3,4$ and 5$)$ were in the invited group, (Chi-squared statistic 709.77, $\mathrm{p}<0.001$ ), with over $80 \%$ of those invited being in step 2 or above.

From evaluable prescribing data, invited patients $(n=5900)$ and those not invited ( $n=6356)$ for clinic review had been prescribed an average of 9.87 (SD 7.81) and 5.25 (SD 6.36) SABA reliever inhalers in the previous 12 months, respectively ( $\mathrm{T}=35.81 ; \mathrm{p}<0.001)$.

1291 (61\%) of the 2123 patients who attended the clinics were using pMDIs, of whom over $80 \%$ were in BTS/SIGN Steps 2 and $3 .^{1}$

\section{pMDI inhaler technique}

Of the 1291 patients using pMDIs, 1275 (99\%), 1207 (93\%), and $528(41 \%)$, had their pMDI technique tested before, once and twice after instruction, respectively (i.e. the first, second and third AIM tests). There were 1092 patients (85.6\%) who failed the first test using the AIM: $946(78.4 \%)$ and 347 $(65.7 \%)$ patients failed the second and third tests.

Tables 1 and 2 show the cross-tabulated results comparing
Table 1. Cross-tabulation of AlM (Vitalograph) test: First versus second test $(p<0.01)$.

\begin{tabular}{l|l|rrr}
\multicolumn{5}{c}{$\begin{array}{c}\text { Second test of inhaler technique } \\
\text { using AlM (Vitalograph) Machine }\end{array}$} \\
\hline & & Fail & Pass & Total \\
\hline First test of inhaler & Fail & 909 & 159 & 1068 \\
technique using & Pass & 28 & 101 & 129 \\
$\begin{array}{l}\text { AlM (Vitalograph) } \\
\text { Machine }\end{array}$ & Total & 937 & 260 & 1197 \\
\hline
\end{tabular}

Table 2. Cross-tabulation of AIM (Vitalograph) test : First versus third test $(p<0.01)$.

\begin{tabular}{|c|c|c|c|c|}
\hline & & \multicolumn{3}{|c|}{$\begin{array}{l}\text { Third test of inhaler technique } \\
\text { using AIM (Vitalograph) Machine }\end{array}$} \\
\hline & 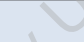 & Fail & Pass & Total \\
\hline First test of inhaler & Fail & 335 & 132 & 467 \\
\hline $\begin{array}{l}\text { technique using } \\
\text { AIM (Vitalograph) }\end{array}$ & Pass & 12 & 49 & 61 \\
\hline Machine & Total & 347 & 181 & 528 \\
\hline
\end{tabular}

the first and second, and first and third, tests after instruction (cross-tabulations show evaluable data available for both of these pairs of patient groups tested). There was a statistically significant increase in the numbers of patients able to use their pMDIs correctly following instruction after the second (129 to 260 of 1197 patients, $p<0.01$ ) and third (61 to 181 of 528 patients, $p<0.01)$ tests. However, on crosstabulation, 323/527 $(61.3 \%)$ of those who were tested after two and three occasions after instruction, failed on both occasions $(p<0.01)$. Over $54 \%$ and $60 \%$ failed the inspiratory flow criterion on the second and third tests, respectively. A logistic regression analysis failed to show any effect of age and BTS step on these outcomes.

\section{Discussion}

The major difficulties faced during the service evaluation were mainly due to incomplete and inconsistent data extracted from the different primary care computer systems used by participating practices. Despite this, and due largely to the substantial numbers of patients seen in the EACS clinics, this service evaluation seems to confirm findings from previous studies $^{5.15}$ that significant numbers of patients are unable to use pMDIs correctly, and that some patients cannot be taught to use a pMDI effectively at all.

Clinicians have been aware for over three decades that the majority of patients cannot use their prescribed pMDIs. ${ }^{16}$ This study strengthens the argument for assessment of inhaler technique with reinforcement at every opportunity, together with education where appropriate. Furthermore, the 
data emphasise the need and ongoing responsibility for healthcare providers to ensure that patients prescribed these inhalers are shown how to use them, and are able to demonstrate their ability to do so.

While we cannot be sure that the results reported here are representative of the asthma population at large, others have demonstrated that the majority of patients cannot use their pMDIs properly and our evidence seems to support that. Furthermore, the fact that the practices and patients in our study were 'self-selected' - in that they accepted invitations to host, and to attend, respectively, the nurse-run clinics - does suggest that these are motivated healthcare professionals and patients who would be expected to be more compliant with asthma guidelines and management. Therefore, we believe we have reported a 'best case' scenario in patients who had probably previously been instructed well in the proper use of their inhalers.

As others have demonstrated, ${ }^{9}$ patients' ability to use pMDIs deteriorates over time, and therefore patients' inhaler technique should be tested and reinforced repeatedly. There are now a number of objective tools - including the AIM used in this study - for assessing patients' inhaler technique, and these are detailed in a paper published recently in this journal. ${ }^{17}$ Furthermore, it is pointless to prescribe medication that patients cannot use - not only in terms of waste of resources, but perhaps more importantly the potential for poor disease control with its subsequent health and economic costs. However, with the data reported here we have not demonstrated a definite link between patients' inability to use inhalers and subsequent poor disease control. Clearly, there is a need investigate this further.

On a positive note, whilst over $85 \%$ of our patients were unable to use their pMDI device correctly at first, we did demonstrate a statistically significant increase in the numbers of patients able to learn the correct technique during the clinic session. It is difficult for clinicians to assess patients' inhaler technique and the AIM unit was used to good effect in a previous study. ${ }^{9}$ In the EACS clinics, the use of the AIM unit provided the nurses and patients with an objective assessment of patients' pMDI technique, which provided valuable information for specific problem-orientated teaching resulting in a reduction in the numbers of those unable to use the device correctly from $85.6 \%$ to $61 \%$. The intervention process therefore had significant positive value. Since the EACS clinics were not repeated in the practices, it is not known whether these patients retained their ability to use their pMDI. However, others have demonstrated that at least half of the patients taught to use their pMDI using the AIM as an educational tool retain their ability to use the pMDI correctly. ${ }^{10}$

Of the three technique measures determined by the AIM, the most common error found in this study was the inspiratory flow generated by the patients. This finding questions the value of simply observing patients' inhaler technique, and suggests that more objective methods for assessing technique should be employed in clinical practice. The AIM is one available tool for testing and training patients in the use of their inhaler, and others have been detailed recently. ${ }^{17}$

A potential study weakness is that we did not standardise the method by which patients were taught to use their inhaler before the second and third AIM tests. However, the nurses were all trained to asthma diploma level, and this includes an assessment of the nurses' ability to teach patients how to use their devices. The nurses used the objective information from the AIM test - and when necessary, the detailed reason(s) why patients failed the test - to direct their instructions. So patient inhaler technique instruction was tailored to the specific needs of the patients who failed, and reinforced by the trained nurses' experience in teaching technique. Therefore, we believe that our conclusion, that many patients could not learn to use pMDIs despite training, is justified.

Another limitation of this study is the potential bias in practice and patient selection. The practices were invited by representatives of Teva UK Limited and the practices themselves decided which patients to invite for asthma review by the trained nurses. The fact that practices accepted the invitation to participate demonstrated a willingness on their part to assess and improve the care of their patients, and the practices we think benefitted from the in-depth discussions on protocols for asthma clinics and also from their deliberations in deciding which patients to review. While the patient selection process used by practices did vary, the overall principles were to identify those patients who could benefit most from an expert assessment - i.e. those with probable poorly controlled asthma. Since about half of the patients seen in the clinics were over 45 years old, it is possible that some would have co-morbid illness such as chronic obstructive pulmonary disease (COPD) - though, as others have emphasised, inhaler technique is also very important in these patients as well. ${ }^{18}$

Prescriptions for SABA inhalers ranged from 0-108 within the past 12 months amongst those patients attending the EACS clinics, and about $50 \%$ of patients had been prescribed four or more inhalers. Two interesting points arise from this data. Firstly, since half of the patients were prescribed no more than four SABA inhalers in 12 months, the practices therefore used additional criteria for selecting patients for review. Indeed, many of the uncontrolled patients eventually selected were apparently not using their SABA inhalers excessively - consistent with the hypothesis that they were symptomatic and warranted review either because they were undertreated and/or they were not taking their prescribed medications. Secondly, sadly, the number of inhalers 
Key Messages

- pMDIs recommended by Primary Care Organisations (PCOs) on the basis of cost alone are not necessarily in the patient's best interest.

- A majority of patients cannot use their pMDI correctly

- The patient should have their ability to use an inhaler device assessed by a competent healthcare professional at every review.

prescribed for some of these patients does call into question the rigour with which prescribing was monitored in these practices, and constitutes a salient lesson for healthcare professionals as well as dispensing pharmacists that more careful monitoring of prescribing should occur.

This service evaluation report has highlighted problems in assessing primary care-run asthma clinics. We identified some of the problems in assessing these and have also confirmed the importance of including inhaler technique checks in this process. High numbers of treated asthma patients are unable to use their medication properly. This experience further emphasises the need for consistent systems for recording routine data in general practice using agreed coding parameters.

\section{Conclusions}

For further evaluation of asthma review services, standardised systems for implementing and assessing the effects of patient education and data recording are needed in routine practice. Despite training, a significant majority of symptomatic asthma patients in this study population were unable to use their prescribed pMDIs correctly. There is therefore a need for appropriately trained practitioners who can assess inhaler technique regularly when reviewing patients with asthma. This should be followed by appropriate action which could include prescription of a different device. Furthermore, cost alone should not determine recommendations for prescribing. Further research is needed to determine the clinical outcomes - particularly the level of asthma control - resulting from patients' inability to use their inhaled medication correctly.

\section{Conflict of interest declarations}

The EACS Service is provided by National Services for Health Improvement (NSHI) and is sponsored by Teva UK Limited.

$\mathrm{JH}$ is an employee of Teva UK Limited, Harlow, Essex

MLL has accepted sponsorship from GlaxoSmithKline (GSK), AstraZeneca (AZ), Boehringer Ingelheim (BI), Cheisi, Merck Sharpe and Dohme (MSD), Merck, Altana Pharma, Novartis, Meda Pharmaceuticals, 3M Pharmaceuticals, and Schering Plough for attending conferences. He has accepted lecture fees from BI, GSK, AZ, Cheisi and Alk-Abello. He has been on advisory boards or provided consultancy for GSK, Schering Plough, MSD, Cheisi, Altana Pharma, Ranbaxy, AZ, 3M Pharmaceuticals and Novartis. He has had research grants from BI, Pfizer and GSK. He is a member of the ADMIT Group, which receives an unrestricted educational grant from MEDA pharmaceuticals. He is the former Editor-in-Chief of the PCRJ, but was not involved in the editorial review of, nor the decision to publish, this article.

\section{References}

1. British Thoracic Society, Scottish Intercollegiate Guidelines Network. British Guideline on the Management of Asthma. Thorax 2008;63(Supplement 4):iv1iv121. [Accessed 18/11/2010] Available from: http://dx.doi.org/ http://www.sign.ac.uk/guidelines/fulltext/101/index.html

2. The Global Strategy for Asthma Management and Prevention, Global Initiative for Asthma (GINA) Updated 2008. 2008 [Last Accessed 18/11/2010]; Available from: http://www.ginasthma.org;

3. Crompton GK. Problems patients have using pressurized aerosol inhalers. Eur J Resp Diseases 1982;63(Suppl.119):101-04.

4. Giraud V, Roche N. Misuse of corticosteroid metered-dose inhaler is associated with decreased asthma stability. Eur Respir J 2002;19(2):246-51. http://dx.doi.org/ 10.1183/09031936.02.00218402

5. Van Beerendonk I, Mesters I, Mudde AN, Tan TD. Assessment of the inhalation technique in outpatients with asthma or chronic obstructive pulmonary disease using a metered-dose inhaler or dry powder device. Journal of Asthma 1998;35(3):273-9. http://dx.doi.org/ 10.3109/02770909809068218

6. Hanania NA, Wittman R, Kesten S, Chapman KR. Medical personnel's knowledge of and ability to use inhaling devices: Metered-dose inhalers, spacing chambers, and breath-actuated dry powder inhalers. Chest 1994;105(1):111-16

7. MIQUEST. [Last Accessed: 4/4/2010]; Available from: http://www.connectingforhealth.nhs.uk/systemsandservices/ssd/prodserv/vapro dmiquest/? searchterm=miquest;

8. Bucknall CE, Ryland I, Cooper A, Coutts, II, Connolly CK, Pearson MG. National benchmarking as a support system for clinical governance. J $R$ Coll Physicians Lond 2000;34(1):52-6.

9. Skaer TL, Wilson CB, Sclar DA, et al. Metered-dose inhaler technique and quality of life with airways disease: Assessing the value of the vitalograph in educational intervention. J Int Med Research 1996;24(4):369-75.

10. Sarvis CA, Tice AL, Plake KS. Evaluation of inhaler technique using the aerosol inhalation monitor. J Pharm Practice 2004;17(4):275-9. http://dx.doi.org/ 10.1177/0897190004270027

11. Nursing and Midwifery Council. Standards of conduct, performance and ethics for nurses and midwives. [Last Accessed: 4/4/2010]; Available from: http://www.nmc-uk.org/aArticle.aspx?ArticlelD=3056;

12 The Association of the British Pharmaceutical Industry (ABPI) Code of Practice. [Last Accessed: 4/4/2010]; Available from: http://www.abpi.org.uk/ publications/pdfs/pmpca_code2008.pdf;

13. The Caldicott Committee. Report on the Review of Patient-Identifiable Information. 1997 [Last Accessed: 4/4/2010]; Available from: http://www.wales.nhs.uk/sites3/Documents/783/DH\%5F4068404\%5B1\%5D.pdf

14. UK Data Protection Act. 1998 [Last Accessed: 4/4/2010]; Available from: http://www.opsi.gov.uk/acts/acts1998/ukpga_19980029_en_1;

15. Virchow JC, Crompton GK, Dal Negro R, et al. Importance of inhaler devices in the management of airway disease. Resp Med 2008;102(1):10-19. http://dx.doi.org/10.1016/j.rmed.2007.07.031

16. Broeders MEAC, Sanchis J, Levy ML, Crompton GK, Dekhuijzen PNR, on behalf of the ADMIT Working Group. The ADMIT series - Issues in Inhalation Therapy. 2) Improving technique and clinical effectiveness. Prim Care Respir J 2009;18(2):76-82. http://dx.doi.org/10.4104/pcrj.2009.00025

17. Lavorini F, Levy ML, Corrigan C, Crompton G, on behalf of the ADMIT Working Group. The ADMIT series - Issues in Inhalation Therapy. 6) Training tools for inhalation devices. Prim Care Respir J 2010;19(4):335-41. http://dx.doi.org/10.4104/pcrj.2010.00065

18. Vincken W, Dekhuijzen PNR, Barnes P, on behalf of the ADMIT Group. The ADMIT series - Issues in Inhalation Therapy. 4) How to choose inhaler devices for the treatment of COPD. Prim Care Respir J 2010;19(1):10-20. http://dx.doi.org/10.4104/pcrj.2009.00062 